\title{
The acceptability of a tailored Mediterranean lifestyle education resource among patients with Mild Cognitive Impairment
}

Andrea M. McGrattan ${ }^{1}$, Claire T. McEvoy${ }^{1}$, Bernadette McGuinness ${ }^{1}$, Michelle C. McKinley ${ }^{1}$, Charlotte E. Neville ${ }^{1}$ and Jayne V. Woodside ${ }^{1}$

${ }^{1}$ Centre for Public Health, Queens University Belfast, BT12 6BA.

\section{BACKGROUND}

Research evidence suggests that greater adherence to a traditional Mediterranean diet (MD) is neuroprotective and may help reduce dementia risk. There is a need to understand how best to achieve behaviour change towards a MD, particularly in non-Mediterranean and high-risk populations, for example, individuals with Mild Cognitive Impairment (MCl). The Theoretical Domains Framework (TDF) (Michie et al., 2014) is based on 14 theoretical domains and is used to help design, develop and deliver behaviour change interventions. We developed a MD educational resource for MCI patients in accordance with the MRC guidance (Craig et al., 2008), based on a systematic literature review and informed by qualitative work (Neville et al., 2013) with the target group. This refined resource was evaluated by structured interviews with $\mathrm{MCl}$ patients using the TDF framework and to further understand the barriers and enablers to dietary change in this patient group.

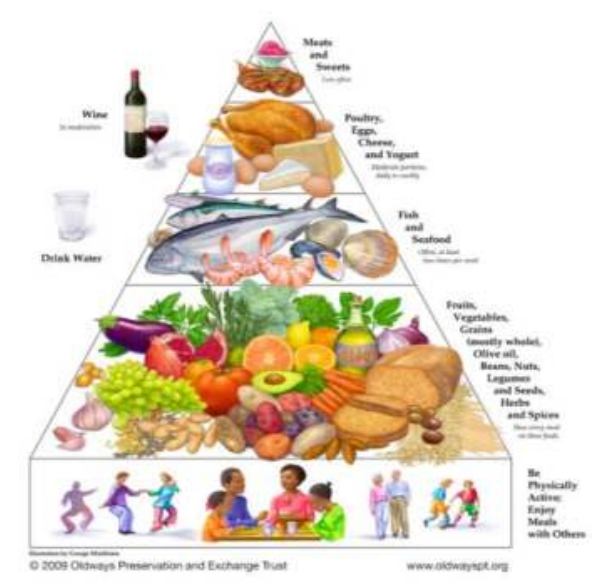

METHODS

$\mathrm{MCl}$ patients were recruited from memory clinics to participate in structured interviews. An interview topic guide was developed using the Theoretical Domains Framework (TDF) (Michie et al., 2014).
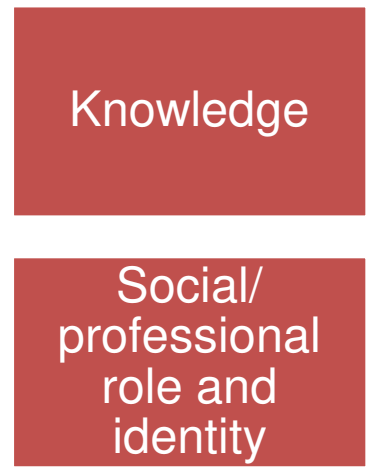
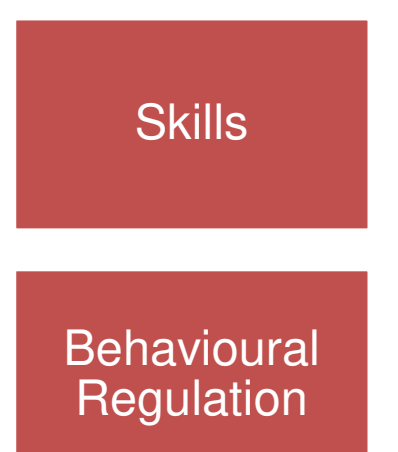

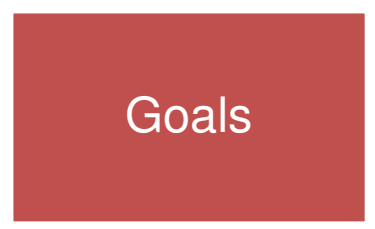

Optimism

Figure 1: Theoretical Domains Framework (TDF) (Michie et al., 2014)

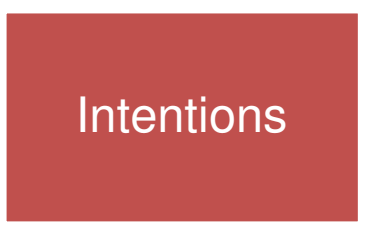

Emotion
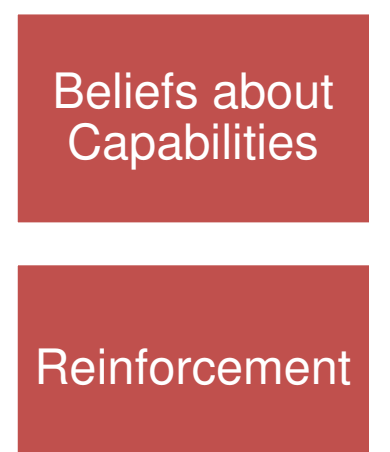
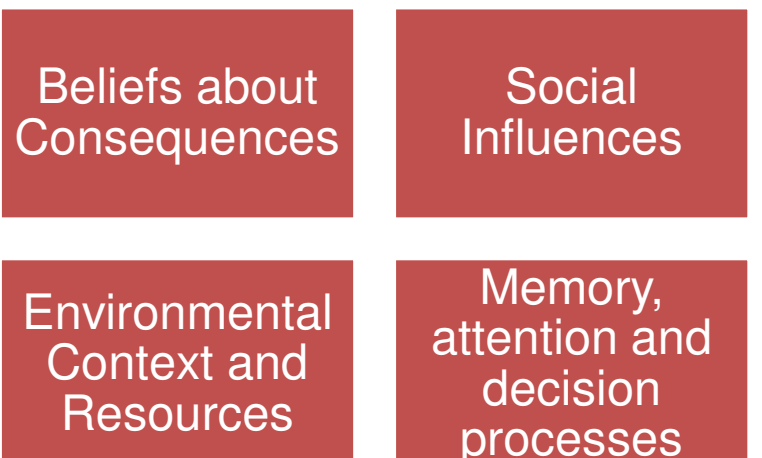

The Mediterranean Lifestyle Education Resource, "THINK-MED” consisted of:

1) Mediterranean Lifestyle Education for Memory - Information Leaflet

2) Four seasonal recipe books

3) 7-day Mediterranean Diet menu plans

4) Mediterranean Lifestyle Education for Memory - Memory Book

5) Calendar

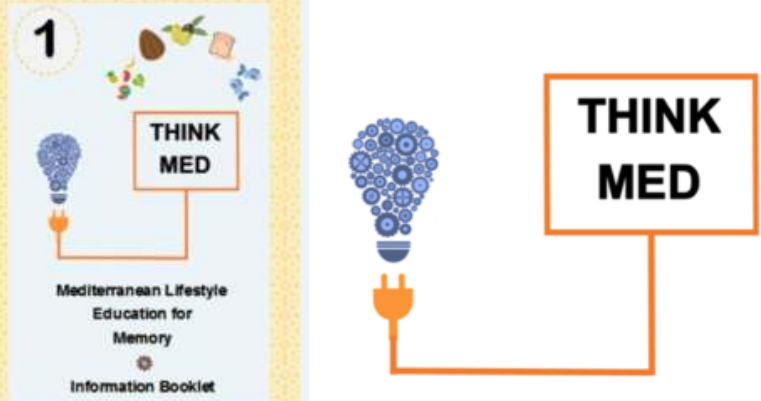

RESULTS AND DISCUSSION

$\mathrm{n}=8 \mathrm{MCl}$ patients (plus two relatives) took part in structured interviews (mean age $=77.0$ years). There were an equal number of male and female participants.
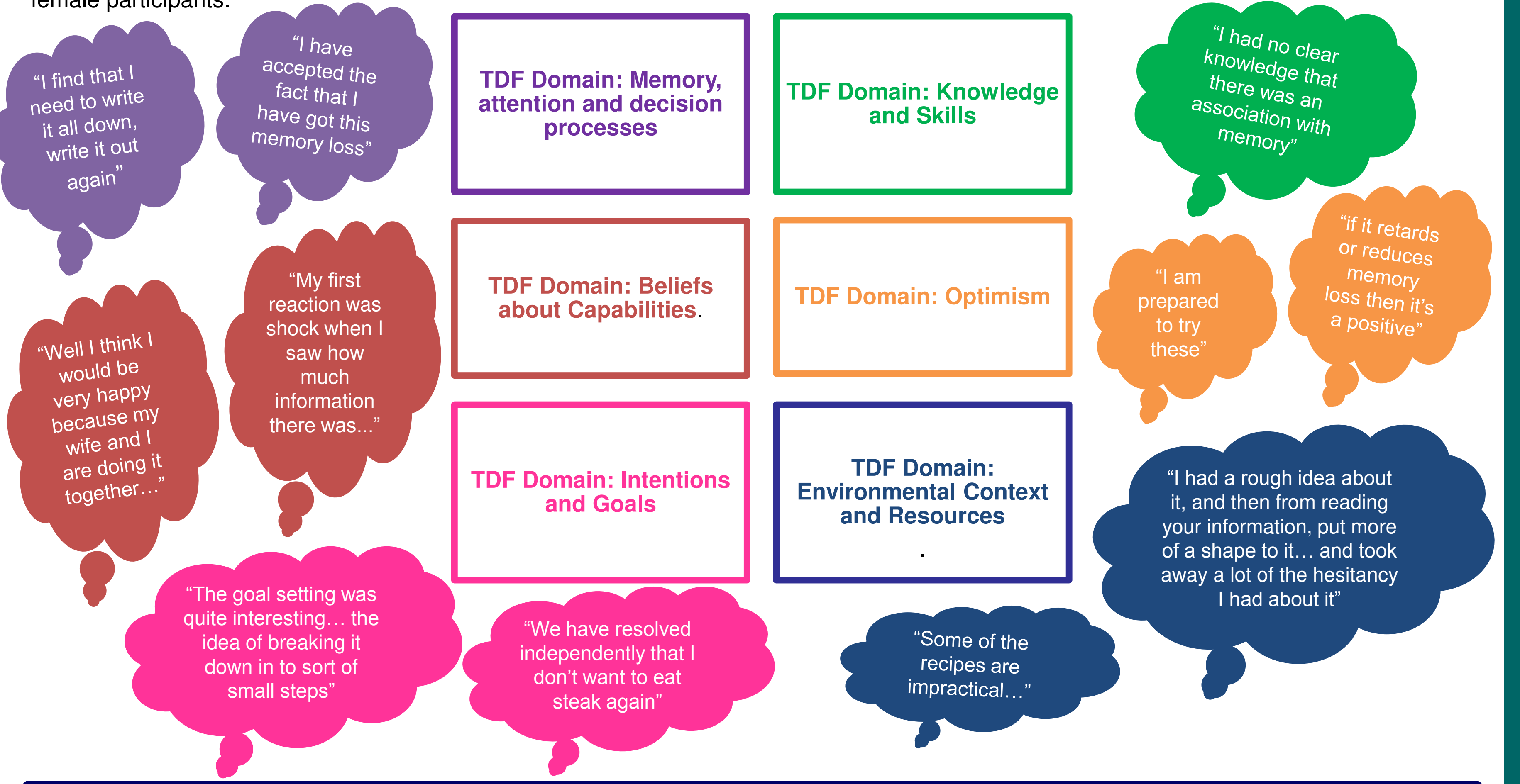

main: Intentions
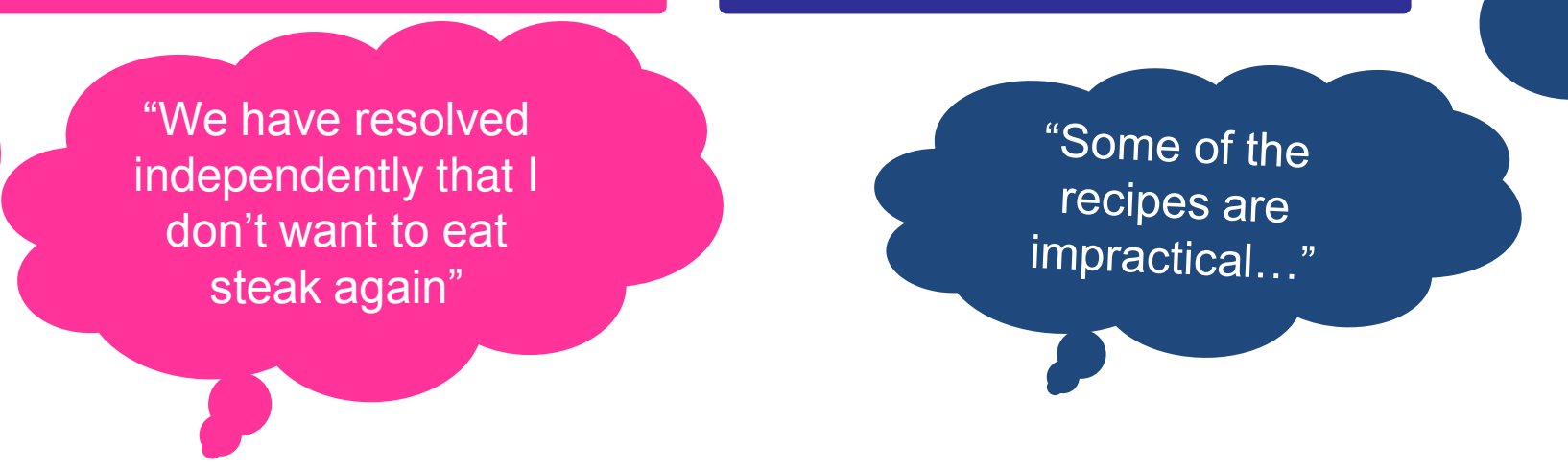

"I had a rough idea about it, and then from reading your information, put more of a shape to it... and took away a lot of the hesitancy I had about it"

\section{CONCLUSION}

Overall, the resource was well received by participants, however discussions highlighted the need to consider adaptions so that the educational resources and associated intervention could be tailored to the needs of an older population.

This information was used to further refine the Mediterranean Lifestyle education resource, "THINK-MED", before its evaluation through a pilot randomised controlled trial, which is currently underway. 